\title{
Postal Code
}

National Cancer Institute

\section{Source}

National Cancer Institute. Postal Code. NCI Thesaurus. Code C25621.

Any system designed to expedite the sorting and delivery of mail by assigning a series of alphanumeric codes to each delivery area. Also used to refer to any individual delivery area code. 